\title{
Dendrite Spacings in Directionally Solidified Superalloy PWA-1480
}

\author{
M. Vijayakumar \\ Cleveland State University \\ Surendra N. Tewari \\ Cleveland State University \\ James E. Lee \\ NAllSA Mthis and additional works at: https://engagedscholarship.csuohio.edu/encbe_facpub \\ Part of the Materials Science and Engineering Commons, and the Thermodynamics Commons \\ P. A. Curreri \\ NASA Mas ancriess to this work benefit you? Let us know! \\ Publisher's Statement
}

NOTICE: this is the author's version of a work that was accepted for publication in Materials

Science and Engineering A. Changes resulting from the publishing process, such as peer review, editing, corrections, structural formatting, and other quality control mechanisms may not be reflected in this document. Changes may have been made to this work since it was submitted for publication. A definitive version was subsequently published in Materials Science and Engineering A, 132, (February 1991) DOI 10.1016/0921-5093(91)90375-W

\section{Original Citation}

Vijayakumar, M., Tewari, S., Lee, J., , \& Curreri, P. (1991). Dendrite spacings in directionally solidified superalloy PWA-1480. Materials Science \& Engineering A, 132, 195-201. doi:10.1016/

0921-5093(91)90375-W

\section{Repository Citation}

Vijayakumar, M.; Tewari, Surendra N.; Lee, James E.; and Curreri, P. A., "Dendrite Spacings in Directionally Solidified Superalloy PWA-1480" (1991). Chemical \& Biomedical Engineering Faculty Publications. 58.

https://engagedscholarship.csuohio.edu/encbe_facpub/58

This Article is brought to you for free and open access by the Chemical \& Biomedical Engineering Department at EngagedScholarship@CSU. It has been accepted for inclusion in Chemical \& Biomedical Engineering Faculty Publications by an authorized administrator of EngagedScholarship@CSU. For more information, please contact library.es@csuohio.edu. 


\title{
Dendrite spacings in directionally solidified superalloy PWA-1480
}

\author{
M. Vijayakumar and S. N. Tewari \\ Chemical Engineering Department, Cleveland State University, Cleveland, OH 44115 (U.S.A.)
}

James E. Lee and P. A. Curreri

NASA-Marshall Space Flight Center, Huntsville, AL 35812 (U.S.A.)

\begin{abstract}
Primary dendrite spacings and side-branch coarsening kinetics were examined in specimens of the single-crystal multicomponent commercial superalloy PWA-1480, which were directionally solidified in a positive thermal gradient. The experimentally observed dependence of primary dendrite spacings and side-branch coarsening kinetics on growth rate and thermal gradient were in agreement with the behavior predicted by analytical models developed for binary alloys.
\end{abstract}

\section{Introduction}

A complex branched dendritic microstructure is commonly produced during solidification of binary alloys. The breakdown of a planar liquid-solid interface first results in the formation of cells, the fingers of the solid protruding into the solidifying melt. These fingers are called primary dendrites when increasing constitutional supercooling in the intercellular melt results in the formation of side branches (secondary arms). Dendrite spacings have a significant influence on mechanical properties. The solidification parameters which control the formation and distribution of primary and secondary dendrites have, therefore, been extensively studied. Several analytical models have been proposed in the literature for predicting the dependence of the primary dendrite spacing $\lambda_{1}$ in binary alloys on the solidification rate $R$, the thermal gradient in the melt at the liquid-solid interface $G_{1}$ and the alloy solute content $C_{0}[1-3]$. Hunt [1], using the approach taken by Bower et al. [4] for modeling microsegregation for a primary dendrite array, obtained the following relationship for $\lambda_{1}$

$$
\lambda_{1}{ }^{2}=4 \sqrt{2}\left\{\left(G_{\mathrm{c}}{ }^{\mathrm{c}} / G_{\mathrm{c}}\right)-1\right\}\left(D_{1} / R\right) r_{\mathrm{t}}
$$

where $D_{1}$ is the solute diffusivity in the melt and $r_{\mathrm{t}}$ is the radius at the tip of the primary dendrite. Neglecting the solutal diffusion in the solid, the concentration gradient in the melt at the dendrite tip $G_{\mathrm{c}}{ }^{\mathrm{t}}$ during steady-state directional solidification is equal to $R C_{1}(k-1) / D_{1}$, where $C_{t}$ is the solute content of the melt at the dendrite tip and $k$ is the solute partition coefficient. Because of the assumptions of local equilibrium and constant thermal gradient, the interdendritic composition gradient $G_{\mathrm{c}}$ is taken to be equal to $G_{1} / m_{1}$, where $m_{1}$ is the liquidus slope. Hunt used $C_{t}$ and $r_{t}$ predictions from the dendrite growth model of Burden and Hunt [5] in the above relationship, and showed that for a variety of binary alloys, the experimentally observed primary arm spacings are proportional to $G_{1}^{-0.5}$ and $R^{-0.25}$, as predicted from eqn. (1). However, detailed quantitative comparisons show that this approach underestimates the experimentally observed primary dendrite spacings $[3,6-8]$. The dendrite growth model of Burden and Hunt [5] treats an array of primary dendrites and assumes minimum undercooling at the tips to predict $r_{\mathrm{t}}$ and $C_{\mathrm{t}}$. Trivedi [3] incorporated the $C_{\mathrm{t}}$ and $r_{\mathrm{t}}$ predictions from his model (which assumes a marginally stable paraboloidal dendrite tip) in eqn. (1) in order to predict dependence of primary dendrite spacings on the thermal gradient and growth rate. This approach shows a good quantitative agreement with the experimental data for succinonitrile-acetone [3] and other binary alloy dendrites [6]. An equally good agreement with 
the experimental data is obtained when eqn. (1) is used with the $r_{\mathrm{t}}$ and $C_{\mathrm{t}}$ predictions from the dendrite growth models of Laxmanan [6]. While extensive $G_{1}, R$ and $\lambda_{1}$ data are available in the literature from directional solidification experiments on binary alloys, where actual thermal gradients were carefully measured by thermocouples placed in the melt, such data for commercial alloys are very limited $[9,10]$. The feasibility of using the above models for predicting quantitatively the experimentally observed primary dendrite spacings in commercial multicomponent alloys is relatively unexplored.

Experiments on directionally solidified alloys have shown that the initial side-branch spacing near the tip of a primary dendrite is about $2-3$ times the dendrite tip radius $[11,12]$. This ratio has been observed to be 3 for succinonitrile [13] and 4.7 for water-ammonium-bromide solution [14], during growth of dendrites in supercooled melts. The spacing between neighboring side branches is observed to increase with increasing distance from the dendrite tip. Several factors have been proposed in the literature which may be responsible for the secondary arm coarsening. The coarsening may be due to simultaneous dissolution of smaller arms with sharper tip radii and growth of larger arms with less curvature $[15$, $16]$. It may be due to the process of dendritic separation, i.e. the secondary arm dissolves and detaches from the primary dendrite (owing to the sharp curvature which reduces the local liquidus temperature) [16]. The coalescence of the neighboring side-branches can also lead to coarsening [17]. Kirkwood [18] has recently proposed a simple analytical model to predict the sidebranch coarsening kinetics in binary alloys. This model is especially suited to partial directional solidification and quenching experiments where measurements of the side-branch spacing from the tip of the primary dendrites to their base in the quenched mushy region of the microstructure can be carried out $[12,18]$. The analytical model assumes a constant temperature and composition of the interdendritic melt. It treats the coarsening process as the simultaneous dissolution of smaller arms (spheres) with sharper tip radii and growth of larger arms with less curvature.

This paper is part of a detailed investigation into the possible effects of reduced convection on microsegregation and dendritic morphologies in directionally solidified single crystals of a nickel base superalloy, PWA-1480. This alloy, with a nominal composition of $\mathrm{Ni}-12 \mathrm{Ta}-10.4 \mathrm{Cr}-5 \mathrm{Co}-$ $5 \mathrm{Al}-4 \mathrm{~W}-1.5 \mathrm{Ti}$ (in weight per cent) is used as single-crystal blades in gas turbine aeroengines. Understanding the dependence of the primary and secondary dendrite spacings and the microsegregation behavior on the processing parameters is of great importance in modeling the formation of solidification-related defects, such as microporosity and macrosegregation, especially freckling. The purpose of this study was to measure the dependence of primary dendrite spacings on thermal gradients and growth speeds, and to explore the feasibility of predicting it quantitatively using the analytical models available for binary alloys. Similar examination of the side-branch coarsening kinetics was the second purpose. This alloy has been selected because the solidification behavior of PWA-1480, despite its being a multicomponent system, is similar to that of a binary alloy. The final solidification at the base of primary dendrites in this alloy occurs in a narrow temperature range (about 5-7 K) [19]; it can be considered nearly isothermal. Unlike other commercial superalloys, it does not form any interdendritic precipitates in the mushy zone. The side branch coarsening analysis is therefore not complicated by the formation of carbide precipitates between the liquidus and eutectic temperatures. In addition, the solutal partition coefficients in PWA-1480 are independent of temperature in the range $1610-1585 \mathrm{~K}$ [19].

\section{Experimental details}

Single-crystal cylindrical specimens (length $7 \mathrm{~cm}$, diameter $0.5 \mathrm{~cm}$ ) of the superalloy PWA1480 , were directionally solidified in alumina crucibles under flowing argon atmosphere in a Bridgman-type furnace. The growth direction was within $\pm 8^{\circ}$ of the $[100]$ orientation of the f.c.c. nickel based gamma matrix in these specimens. Details of the directional solidification furnace are presented in ref. 20. Directional solidification was carried out by translating the furnace (gradient zone) at constant speeds varying from 0.001 to $0.023 \mathrm{~cm} \mathrm{~s}^{-1}$. Two $\mathrm{Pt}-\mathrm{Pt}-$ $13 \% \mathrm{Rh}$ thermocouples kept inside the specimen (located along the specimen length with a distance of about $0.5 \mathrm{~cm}$ between thermocouple beads) were used to record the in situ thermal profile. When the thermocouple extending farther into the melt was at a predetermined temperature, usually near the liquidus temperature of 
the alloy, $1610 \mathrm{~K}$, the furnace was quickly withdrawn, positioning a quench nozzle over the solid-liquid zone and quenching by spraying water on the crucible surface.

The specimens were metallographically polished and etched (33 acetic acid, 33 nitric acid, 33 water and 1 hydrofluoric acid by volume) to correlate microstructural features, such as the tip and base of primary dendrites, with the temperatures measured by the two thermocouples. The dendrite tip radius was measured for one sample by serial sectioning of the primary dendrites on longitudinal sections (parallel to the alloy growth direction), and fitting a parabola to the midsection of the dendrite near its tip. Primary dendrite spacings were measured on transverse sections (perpendicular to the growth direction). The spacings reported in this paper are equal to $(A / N)^{0.5}$, where $A$ is the total specimen crosssection area (transverse section) and $N$ is the number of primary dendrites on that crosssection. Depending on the growth conditions, 200-600 primary dendrites were observed and counted on the corresponding specimen crosssections $\left(18.58-20.14 \mathrm{~mm}^{2}\right)$.

Secondary arm spacings were measured by averaging the distance between five adjacent side branches on the longitudinal section (parallel to the alloy growth direction) of a primary dendrite as a function of distance from the dendrite tip. Each of the side branch spacing data reported here is the average of secondary arm spacings from four or five primary dendrites. The main source of scatter in this method of obtaining the secondary arm spacing data is the misalignment of primary dendrites, in the mushy region, with respect to the growth direction (growth direction is in the plane of metallographic polishing). This causes larger scatter in samples with thinner primary dendrites, i.e. those solidified at higher growth speeds.

Two experimental observations were used to ascertain that the steady-state growth conditions were achieved before the specimen was quenched: the variation in primary dendrite spacings along the length of the directionally solidified sample and the thermal profiles from the two thermocouples. Primary and secondary arm spacings reported in this paper correspond to the specimen portions where primary arm spacings were constant along the specimen length. In this portion of the sample, the thermal gradients measured at the tips of primary dendrites $G_{1}$, assumed to be at the alloy liquidus temperature $(1610 \mathrm{~K})$, from the two thermocouples were within $\pm 6 \%$. The growth speed is therefore assumed to be identical with the furnace translation speed in the present analysis. The side branch coarsening time $t$ was obtained as the ratio of the distance from the quenched primary dendrite tips (the liquidus temperature) along the specimen length in the mushy region and the growth speed. Table 1 lists the growth parameters of the specimens examined in this study. A cooling rate typically about $50 \mathrm{~K} \mathrm{~s}^{-1}$ was recorded during quenching by the thermocouple located near the dendrite tips.

\section{Results and discussion}

Figure $1(\mathrm{a})$ shows a typical longitudinal section (parallel to the growth direction) through a directionally solidified specimen. This specimen was solidified at a growth speed of $0.013 \mathrm{~cm} \mathrm{~s}^{-1}$ $\left(G_{1}=170 \mathrm{~K} \mathrm{~cm}^{-1}\right)$. As mentioned earlier, the temperatures shown in this figure were obtained by correlating the location of the thermocouple tips in the longitudinal microstructures and the corresponding thermal profiles. The use of single-crystal specimens has resulted in primary dendrites having excellent alignment with the growth direction. This reduces the contribution to the experimental scatter in the dendrite spacings from their poor alignment in the microstructure. A distinct change in the microstructure of the quenched interdendritic region, observed at a

\section{TABLE}

Effect of the thermal gradient and growth rate on the primary arm spacing of directionally solidified superalloy PWA-1480

\begin{tabular}{cllll}
\hline Sample & $\begin{array}{l}R \\
\left(\mathrm{~cm} \mathrm{~s}^{-1}\right)\end{array}$ & $\begin{array}{l}G_{1} \\
\left(\mathrm{~K} \mathrm{~cm}^{-1}\right)\end{array}$ & $\begin{array}{l}\lambda_{1} \\
(\mathrm{~cm})\end{array}$ & $\begin{array}{l}\text { Cell length } \\
(\mathrm{cm})\end{array}$ \\
\hline $4 \mathrm{G}$ & 0.0033 & 120 & 0.0273 & \\
$9 \mathrm{G}$ & 0.0100 & 85 & 0.0215 & \\
$11 \mathrm{G}$ & 0.0133 & 90 & 0.0219 & \\
$11 \mathrm{~K}$ & 0.0233 & - & 0.0152 & \\
$12 \mathrm{G}$ & 0.0017 & 100 & 0.0335 & \\
$13 \mathrm{G}$ & 0.0010 & 120 & 0.0368 & 0.605 \\
$10 \mathrm{G}$ & 0.0067 & 120 & 0.0245 & 0.581 \\
$1 \mathrm{~K}$ & 0.0233 & 140 & 0.0138 & 0.482 \\
$8 \mathrm{~K}$ & 0.0233 & 150 & 0.0132 & 0.447 \\
$25 \mathrm{G}$ & 0.0233 & 180 & 0.0142 & \\
$13 \mathrm{~K}$ & 0.0133 & 150 & 0.0161 & 0.424 \\
$17 \mathrm{~K}$ & 0.0133 & 170 & 0.0146 & 0.425 \\
$27 \mathrm{G}$ & 0.0133 & 110 & 0.0185 & \\
\hline
\end{tabular}



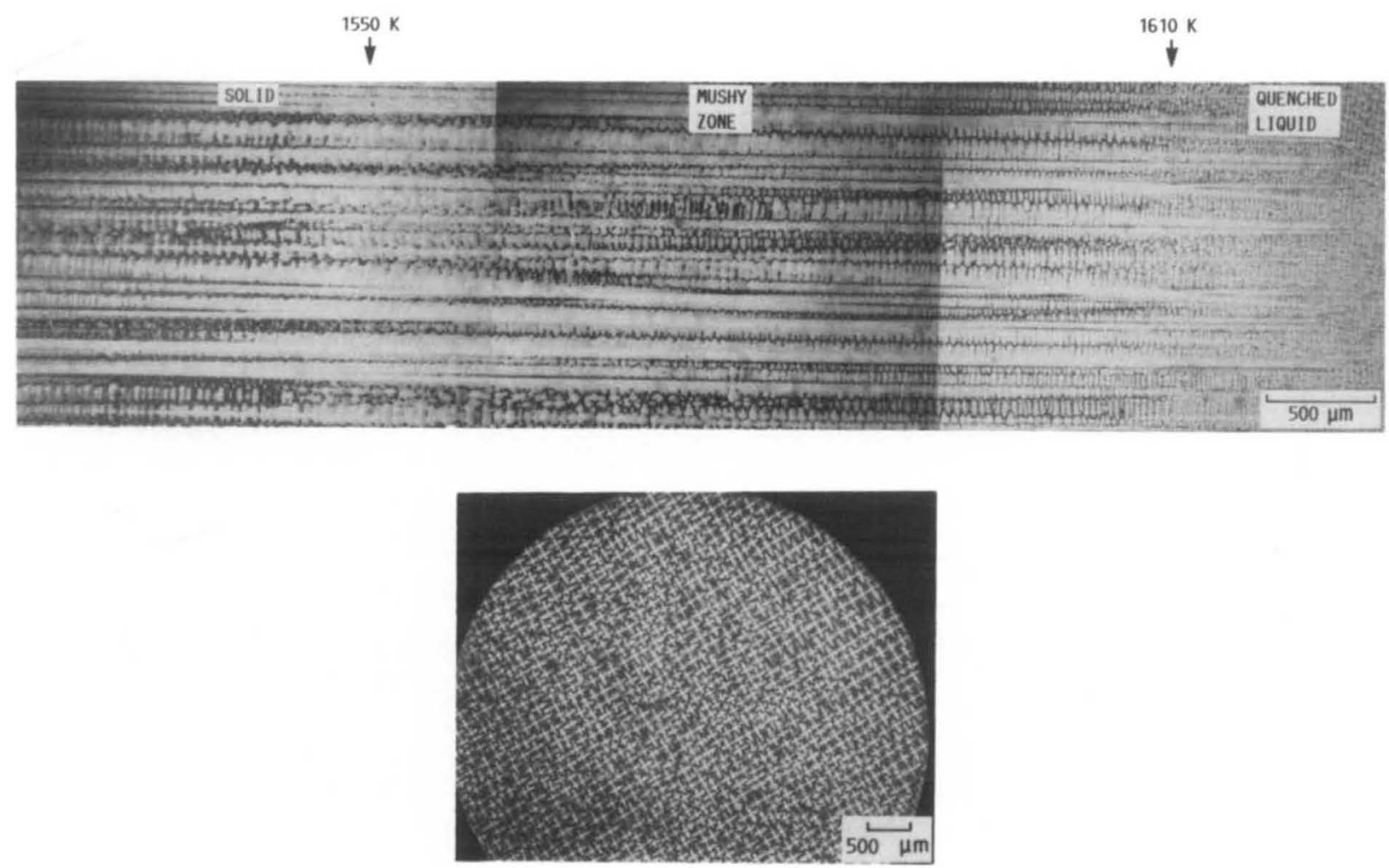

Fig. 1. Microstructure of a directionally solidified superalloy PWA-1480 sample (17K), growth speed $0.013 \mathrm{~cm} \mathrm{~s}^{-1}, G_{1}=170 \mathrm{~K}$ $\mathrm{cm}^{-1}$ : (a) longitudinal section through the quenched mushy zone; (b) transverse section in the directionally solidified portion.

temperature corresponding to about $1550 \mathrm{~K}$ [19], was used to locate the base of the primary dendrite array, where the last interdendritic liquid solidified just before quench. The experimentally observed lengths (cell length) of the primary dendrite arrays protruding into the melt, varying from 0.45 to $0.6 \mathrm{~cm}$, are also listed in Table 1 . Figure 1(b) shows a typical transverse microstructure in the directionally solidified specimen. As seen in this figure, the distribution of primary dendrites was observed to be very uniform across the entire specimen cross-section.

\subsection{Primary dendrite spacings}

At high constitutional supercooling gradients, i.e. for growth conditions where $G_{c}{ }^{t}$ is much larger than $G_{\mathrm{c}}$ or $G_{1} / m_{1}$, the dendrite tip radius predictions from dendrite growth models for binary alloys are nearly the same $[6,21]$. Incorporation in eqn. (1) of the dependence of the tip radii on the growth rate and thermal gradient, as predicted from these models, yields the following relationship for the primary dendrite spacings

$\lambda_{1}=\left(B 64 \sigma D_{1} k \Delta T_{0} / \Delta S\right)^{0.25} G_{1}^{-0.5} R^{-0.25}$ where $\sigma$ is the liquid-solid surface energy, $\Delta T_{0}=m_{1} C_{0}(k-1) / k$ is the alloy freezing range, $\Delta S$ is the entropy of fusion per unit volume and $B$ is a constant. The value of $B$ is respectively 1,16 or 28 for the dendrite growth models of Burden and Hunt [5], Laxmanan [21] and Trivedi [3]. As mentioned earlier, despite its multicomponent nature, PWA-1480 shows a solidification behavior which is similar to a binary alloy. We will therefore compare the experimentally observed primary arm spacing data with those predicted from analytical models, even though the models are strictly only valid for simple binary alloys directionally solidified in a positive thermal gradient.

Figure 2 shows the dependence of the primary dendrite spacings $\lambda_{1}$ on the thermal gradient $G_{1}$ and growth rate $R$ in directionally solidified single-crystal specimens of PWA-1480. The abscissa in this figure was selected to be $R^{-0.25} G_{1}^{-0.5}$, with $R$ measured in centimeters per second, and $G_{1}$ in degrees Kelvin per centimeter, as suggested by eqn. (2). The solid line in this figure, described by the following relationship, is the linear least-squared fit to the data 


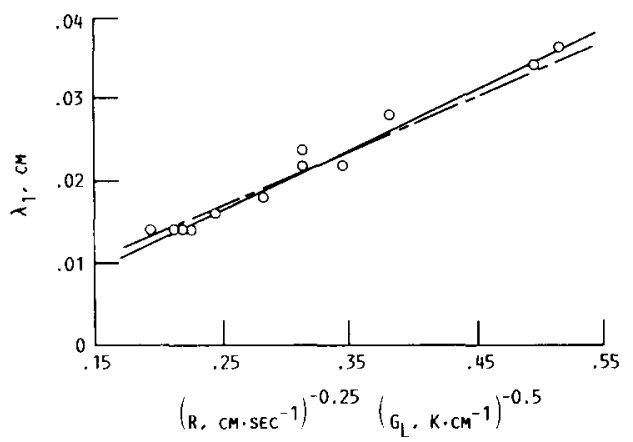

Fig. 2. Dependence of primary dendrite spacings $\lambda_{1}$ on the thermal gradient $G_{1}$ and growth rate $R$ in directionally solidified single-crystal PWA-1480. The solid line is the linear least-squared fit to the experimental data. The broken line is the prediction from Trivedi's model [3].

$$
\begin{aligned}
\lambda_{\mathrm{J}}= & (-0.00128 \pm 0.0013) \\
& +(0.0728 \pm 0.0040) R^{-0.25} G_{1}^{-0.5}
\end{aligned}
$$

where $\lambda_{1}$ is in centimeters, $R$ in centimeters per second, and $G_{1}$ in degrees Kelvin per centimeter. A high value of correlation coefficient, 0.94 , and very low value of relative standard deviation for this fit, $3 \%$, denote an excellent fit of the experimental data to the above linear relationship. The linear dependence of primary dendrite spacings on $G_{1}{ }^{-0.5} R^{-0.25}$, predicted by Hunt's model, is also valid for our complex multicomponent superalloy, PWA-1480.

We will now estimate the solutal diffusivity in the melt by equating $\left(B 64 \sigma D_{1} k \Delta T_{0} / \Delta S\right)^{0.25}$ (eqn. (2)) to the experimental value of the slope, 0.0728 (eqn. (3)). The alloy freezing range, $\Delta T_{0}$ is $35 \mathrm{~K}$ (for PWA-1480 liquidus $1610 \mathrm{~K}$, and solidus $1575 \mathrm{~K})$. The value of $\sigma$ and the heat of fusion per unit volume of PWA-1480 is assumed to be the same as that of nickel, $255 \mathrm{erg} \mathrm{cm}^{-2}$ [22] and $2.77 \times 10^{10}$ erg $\mathrm{cm}^{-3}$ [23] respectively. The entropy $\Delta S$ value used here is that calculated at the alloy liquidus temperature, $1610 \mathrm{~K}$. For $B=28$, as used in Trivedi's model [3], the $D k$ value thus obtained is $3.0 \times 10^{-5} \mathrm{~cm}^{2} \mathrm{~s}^{-1}$. The experimentally observed partition coefficients for the various solutes in superalloy PWA-1480 are in the range 0.4-1.7 [19]. Thus the solute diffusivity in the melt $D_{1}$ is predicted to be in the range $1.8-7.0 \times 10^{-5} \mathrm{~cm}^{2} \mathrm{~s}^{-1}$. This is in reasonable agreement with the reported solutal diffusivity, about $2-8 \times 10^{-5} \mathrm{~cm}^{2} \mathrm{~s}^{-1}[9]$, obtained from estimations based on the use of the constitutional supercooling criterion for plane front solidification of several superalloys. Based on these physical parameters, the primary dendrite spacings predicted from eqn. (2) are plotted in Fig. 2. The broken line in Fig. 2 represents the values predicted from the approach used by Trivedi [3] for $D_{1}=8 \times 10^{-5} \mathrm{~cm}^{2} \mathrm{~s}^{-1}$ and $k=0.6$ corresponding to tantalum in PWA-1480 [19]. It should also be mentioned that the predictions obtained by combining the models of Laxmanan [21] and Hunt [1] are also approximately similar (within $15 \%$ of the values indicated by the broken line in Fig. 2). Considering the uncertainties in the various physical properties and the assumption of treating PWA-1480 as a binary alloy, the experimental data are in good quantitative agreement with the predictions from the analytical models

A comparison of the experimentally observed tip radius of $12 \mu \mathrm{m}$ for one specimen, $G_{1}=120 \mathrm{~K}$ $\mathrm{cm}^{-1}, R=0.001 \mathrm{~cm} \mathrm{~s}^{-1}$, with predictions from dendrite growth models also shows a good agreement. The tip radius value predicted from Trivedi's model [3] is $7.2 \mu \mathrm{m}$ for $D_{1}=2 \times 10^{-5} \mathrm{~cm}^{2}$ $\mathrm{s}^{-1}$ and $14.4 \mu \mathrm{m}$ for $D_{1}=8 \times 10^{-5} \mathrm{~cm}^{2} \mathrm{~s}^{-1}$.

\subsection{Side-branch coarsening kinetics}

In the following, the experimentally observed side-branch coarsening behavior for the superalloy PWA-1480 will be compared with predictions from Kirkwood's analytical model [18], treating the multicomponent superalloy as if it were a binary alloy. As mentioned earlier, this model is especially suited to partial directional solidification and quenching experiments. The model predicts the following behavior:

$d^{3}=\left\{128 \sigma(T / H)\left(D_{1} / k \Delta T_{0}\right)\right\} t$

where $d$ is the side-branch spacing corresponding to the coarsening time $t$ and $H / T$ is the entropy of fusion per unit volume.

Figure 3(a) plots $\log _{10}(d)$ vs. $\log _{10}(t)$ data obtained from several experiments. The corresponding growth speeds are indicated in the figure. The straight line drawn through the data is the linear least-squared fit. The fit is given as

$$
\begin{aligned}
\log _{10}(d)= & (1.021 \pm 0.014) \\
& +(0.33 \pm 0.001) \log _{10}(t)
\end{aligned}
$$

where $d$ is measured in micrometers and $t$ in seconds.

There is considerable scatter in the data, but the experimentally observed slope of the curve, 0.33 , is in agreement with that expected from the above coarsening relationship, eqn. (4). The same 


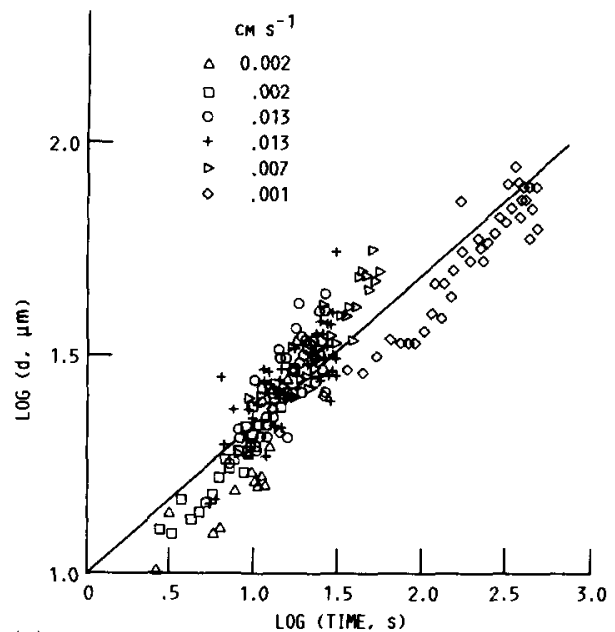

(a)

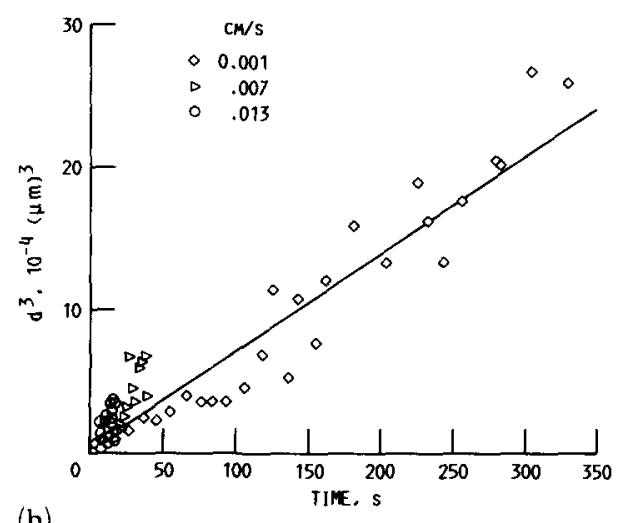

(b)

Fig. 3. Side-branch coarsening kinetics in superalloy PWA1480 , the line is the linear least squared fit through the data: (a) $\log -\log$ plot of side branch spacing $d v s$. coarsening time $t$; (b) plot of the cube of the side branch spacing $d v s$. the coarsening time $t$.

data can also be presented as a linear plot of $d^{3}$ $v s$. $t$, as shown in Fig. 3(b). However, such representation is dominated solely by the slowest growth rate experiment, because of its longest coarsening time. Such is not the case with the $\log -\log$ plot. The linear least-squared fit to the data in Fig. $3(\mathrm{~b})$ is given as $10^{-4} d^{3}=(0.43 \pm 0.21)$ $+(0.075 \pm 0.003) t$. The experimentally obtained value, $1.2 \times 10^{-9} \mathrm{~cm}^{3} \mathrm{~s}^{-1}$, of the terms in the bracket in eqn. (4) (obtained from the intercept on the $Y$-axis in Fig. 3(a)), can be used to obtain the expected $D_{1}$ value for PWA-1480. We will use the physical property values mentioned earlier and assume the coarsening temperature $T$ to be the liquidus temperature, $1610 \mathrm{~K}$. The $\left(D_{1} / k\right)$ value thus obtained by fitting the abovementioned coarsening relationship to the experimental data is about $2 \times 10^{-5} \mathrm{~cm}^{2} \mathrm{~s}^{-1}$. Using the experimentally observed partition coefficients for the various solutes in superalloy PWA-1480, ranging from 0.4 to 1.7 [19] the solute diffusivity $D_{1}$ in the PWA-1480 melt would be predicted to be in the range $0.8-3.4 \times 10^{-5} \mathrm{~cm}^{2} \mathrm{~s}^{-1}$. Considering the uncertainties in the various physical properties, and the assumption of treating PWA-1480 as a binary alloy, this is in reasonable agreement with the value of $1.8-7.0 \times 10^{-5} \mathrm{~cm}^{2} \mathrm{~s}^{-1}$ obtained earlier from examining the dependence of the primary dendrite spacings on the growth rate and thermal gradient.

\section{Conclusions}

The following conclusions can be drawn from this study on single-crystal nickel base superalloy PWA-1480 specimens directionally solidified in a positive thermal gradient. In this study the primary dendrite spacings and side-branch coarsening kinetics were investigated. The alloy PWA-1480, nominal composition $\mathrm{Ni}-12 \mathrm{Ta}-$ $10.4 \mathrm{Cr}-5 \mathrm{Co}-5 \mathrm{Al}-4 \mathrm{~W}-1.5 \mathrm{Ti}$ (weight per cent), has been treated as a simple binary alloy for comparison of the experimental data with predictions from analytical models.

(1) The dependence of the primary dendrite spacing $\lambda_{1}$ on the growth rate $R$ and thermal gradient $G_{1}$ is given as:

$$
\begin{aligned}
\lambda_{1}= & (-0.00128 \pm 0.0013) \\
& +(0.0728 \pm 0.0040) R^{-0.25} G_{1}^{-0.5}
\end{aligned}
$$

with $\lambda_{1}$ measured in centimeters, $R$ in centimeters per second, and $G_{1}$ in degrees Kelvin per centimeter. This relationship is the same as that predicted from the analytical model developed by Hunt [1] for binary alloys. A reasonable quantitative agreement is obtained between the experimental data and the predictions from the analytical model of Trivedi [3].

(2) The secondary arm coarsening kinetics in PWA-1480 obeys the following relationship

$$
\begin{aligned}
\log _{10}(d)= & (1.021 \pm 0.014) \\
& +(0.33 \pm 0.001) \log _{10}(t)
\end{aligned}
$$

where $d$ (in micrometers) is the side-branch spacing corresponding to the coarsening time $t$ (in seconds). This behavior is in agreement with predictions from the model of Kirkwood [18] developed for binary alloys. 


\section{Acknowledgments}

This work was funded by the Marshall Space Flight Center, Center Director's Discretionary Fund, CDDF-87-14, under a cooperative agreement (NAG 8-091). Research facilities in the Microgravity Materials Science Laboratory, NASA-Lewis Research Center, Cleveland, were used during this study. Appreciation is expressed to R. E. Shurney of the Marshall Space Flight Center, G. Workman, G. A. Smith, and R. Bond of the University of Alabama in Huntsville, and the personnel of the Johnson Space Center who operate the $\mathrm{KC}-135$.

\section{References}

1 J. D. Hunt, Solidification and Casting of Metals, Book 192. The Metals Society, London, 1979, pp. 3-9.

2 W. Kurz and D. J. Fisher, Acta Metall., 29 (1981) 11-22.

3 R. Trivedi, Metall. Trans. A, 15 (1984) 977-982.

4 T. F. Bower, H. D. Brody and M. C. Flemings, Trans. Am. Inst. Min. Eng., 236 (1966) 624-634.

5 M. H. Burden and J. D. Hunt, J. Cryst. Growth, 22 (1974) 99-108.

6 S. N. Tewari and V. Laxmanan, Acta Metall., 35 (1987) 175-183.

7 Y. Miyata, T. Suzuki and J. I. Uno, Metall. Trans. A, 16 (1985) 1799-1805.

8 A. Geying and L. Lixin, J. Cryst. Growth, 80 (1987) 383-392.
9 P. N. Quested and M. McLean, Mater. Sci. Eng., 65 (1984) 171-180.

10 S. N. Tewari and A. M. Sriramamurthy, Metall. Trans. A, I2(1980) 137-138.

11 K. Somboonsuk, J. T. Mason and R. Trivedi, Metall. Trans. A, 15(1984) 967-975.

12 S. N. Tewari, V. V. Nesarikar and D. Lee, Metall. Trans. A, 20(1989) $1889-1893$.

13 S. C. Huang and M. E. Glicksman, Acta Metall., 29 (1981) 701-715.

14 H. Honjo and Y. Sawada, J. Cryst. Growth, 58 (1982) 297-303.

15 T. Z. Kattamis, J. C. Coughlin and M. C. Flemings, Trans. Am. Inst. Met. Eng., 239 (1967) 1504-1511.

16 M. Kahlweit, Scr. Metall., 2 (1968) 251-254.

17 K. A. Young and D. H. Kirkwood, Metall. Trans. A, 6 (1975) 197-205.

18 D. H. Kirkwood, Mater. Sci. Eng., 73 (1985) L1-L4.

19 S. N. Tewari, M. Vijayakumar, J. E. Lee and P. A. Curreri, Dendritic morphology and microsegregation in directionally solidified superalloy, PWA-1480, single crystal: effect of gravity, NASA Contract Report, Marshall Space Flight Center, Alabama, 1990, in the press.

$20 \mathrm{G}$. A. Smith and G. Workman, Operational procedure and specifications manual for the $\mathrm{KC}-135$ automatic directional solidification furnace, NASA Contract Report NAS-8-34530, Marshall Space Flight Center, Alabama, 1985.

21 V. Laxmanan, J. Cryst. Growth, 83 (1987) 391-402.

22 J. H. Holloman and D. Turnbull, Progr. Met. Phys., 4 (1953) 333-388.

23 G. H. Geiger and D. R. Poirier, Transport Phenomena in Metallurgy, Addison-Wesley, Reading, MA, 1972, p. 580. 